\title{
ASYNCHRONOUS AND VARIABLE DATA RATE OFDM MODEM FOR RADAR DATA TRANSMISSION
}

\author{
S. Zazo*, I. Raos*, J. López-Pérez ${ }^{\dagger}$, I. Pérez-Álvarez ${ }^{\dagger}$, B. Pérez-Díaz ${ }^{\dagger}$, E. Jiménez-Yguacel ${ }^{\dagger}$ \\ * Universidad Politécnica de Madrid (UPM). Dpto. SSR. ETSI de Telecomunicación. Madrid. Spain. Tel.: +34-915495700 \\ Ext 4042, email: santiago@gaps.ssr.upm.es \\ ${ }^{\dagger}$ Universidad de Las Palmas de Gran Canaria(ULPGC). CeTIC. Dpto. Señales y Comunicaciones. Las Palmas (Canary \\ Islands). Spain. Tel: +34-928457362, email: jlopez@cetic.eu
}

Keywords: RADAR, OFDM, Variable rate, frequency diversity, asynchronous communication.

\begin{abstract}
This paper addresses the real trials of a new OFDM modem specifically designed for data RADAR transmission that was promoted by the Spanish aeronautical authorities. The nature of this environment is very different of standard applications and has required some new features that will be described in the sequel. The data source is asynchronous depending on the appearance of new plots, and therefore the data rate is also variable. Frames transmission must be very short, less than one second because it is a critical real time application. It is unidirectional, that means that we do not have a feedback channel to inform the transmitter about the channel state. To cope with this lack of time diversity we have implemented a parallel transmission strategy by different channels well separated in frequency. Also, FEC and CRC codes are included working with very short interleavers due to the delay requirement. This design has been tested in a real link between Madrid and Las Palmas de Gran Canaria with quite satisfactory performance using our own broadband transceiver to make the multiband transmission efficiently.
\end{abstract}

\section{Introduction}

There are several RADAR systems to control the Atlantic air traffic that are spread over a large area. The information retrieved by these systems must be provided to the control centre in a very short time typically using a set of satellite transponders to include redundancies. This infrastructure guarantees the required quality of service but it is expensive. The Spanish aeronautical service provider (AENA) has promoted a research line in order to check the reliability of a HF link for this purpose as a back-up of the satellite link to reduce the number of required hired bandwidth. The main requirement of the application is that maximum delay should be less than two seconds. However, the acceptable bit error rate (BER) is not so critical because this information is highly correlated in time and space (plots follow well defined tracks in civil applications and the RADAR antenna points to every aircraft in every turn).

The maximum acceptable delay has limited the frame length to one second forcing a very short burst link. The procedure is as follows: when a plot is generated by the source, we store the next plots during one second. At that time the frame is transmitted adding the corresponding header. At the receiver side, we have to wait until the reception of the whole frame to de-interleave and decode the data.

Secondly, it is clear that data rate is variable depending on the air traffic activity. Our design includes the possibility of transmitting 4-QAM, 16-QAM and even 64QAM and also has variable coding rate using a variable shortened Reed-Solomon (and a fixed $1 / 2$ LDPC) to maximize the redundancy. Data interleaving within the frame length is also implemented.

In order to maximize the usage of the less dense constellation (4QAM) according to the statistics of the required data rate of a real RADAR, we have designed the OFDM system to include 150 data carriers in a bandwidth of $6.7 \mathrm{KHz}$. This proposal clearly differs to the standard around $3 \mathrm{KHz}$ channelization and has required the usage of our own transceiver that is flexible to transmit arbitrary bandwidth even in several subbands [6].

First tests using a channel simulator showed that the performance of the coding strategy was successful for most of the situations but there were several frames with long errors burst that degraded the performance in an unacceptable way. As delay was mandatory we have proposed to make a transmission in parallel using different bands in order to retrieve frequency diversity and overcome in some sense the lack of time diversity and improving the performance. This strategy has been possible using our transceiver that is able to transmit a multiband signalling quite efficiently. Simulated and real links have shown that this approach is really critical in order to fulfil the requirements.

Retrieval of the frequency diversity has been implemented by the selection of the plot using the CRC information as a flag of correctness. Other strategies based on MRC principles has been used but were rejected because the improvement was less than expected. This part will be discussed in the sequel.

We have defined the concept of ALMA Tx/Rx as an enhanced Automatic Link MAnager that optimizes the signal and radio resources at both ends.

The feasibility of this approach has been tested in a real link between Madrid and Las Palmas de Gran Canaria separated around $1800 \mathrm{Km}$ during different days and hours testing the effect of the frequency separation in the multiband transmission. 


\section{RADAR data characteristics}

The Spanish Aeronautical service provider (AENA) has provided real data obtained from several operating RADARs from Spain and Portugal. The plot information follows the ASTERIX format [1-3]. Our first task was to evaluate the time-varying activity of these RADARs along different days, changing the possible integration delay. This interval defines the amount of data we store before transmitting a frame. We have considered several study cases with $1,2,4,5$ and 10 seconds. The longer the time, the more uniform the data rate per frame. Next table shows, as an example, these figures for the Spanish RADAR E194 [4], November the $18^{\text {th }}, 2007$.

\begin{tabular}{|l|c|c|c|c|c|c|}
\hline $\begin{array}{l}\text { RADAR } \\
\text { E194 }\end{array}$ & $1 \mathrm{sec}$ & $2 \mathrm{sec}$ & $3 \mathrm{sec}$ & $4 \mathrm{sec}$ & $5 \mathrm{sec}$ & $10 \mathrm{sec}$ \\
\hline $\begin{array}{l}\text { Average } \\
\text { data rate b/s }\end{array}$ & 1085.7 & 1089.5 & 1090.9 & 1091.4 & 1091.9 & 1092.7 \\
\hline $\begin{array}{l}\text { Maximum } \\
\text { Data rate b/s }\end{array}$ & 5728 & 4048 & 3224 & 2674 & 2636.8 & 2432.8 \\
\hline $\begin{array}{l}\text { Average } \\
\text { num. bits }\end{array}$ & 1075.7 & 2178.9 & 3273 & 4366 & 5459 & 10927 \\
\hline $\begin{array}{l}\text { Maximum } \\
\text { Num. bits }\end{array}$ & 5728 & 8096 & 9672 & 10696 & 13184 & 24328 \\
\hline
\end{tabular}

Table 1. RADAR E-194. Analysis of activity. 2007,Nov, $18^{\text {th }}$

Clearly we observe that when decreasing the frame interval, the maximum data rate increases. When increasing the frame interval, the maximum data rate closes the average. However, AENA set up that two seconds was the maximum acceptable delay, therefore the frame length was limited to one second, splitting the available time, one second for storing and coding and other for decoding. Figure 1 shows this scheme where $T_{I}$ means the integration time, $T_{p}$ is some gap, $T_{c}$ the header time, $T_{d}$ the data interval and $T_{f}$ the indicator of the end of the frame.

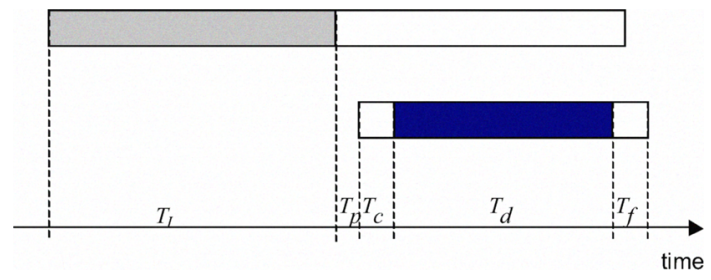

Figure 1. Time structure

Next table shows a summary of the average / maximum data rate considering different days and RADARs [4] for the required 1 second frame length.

\begin{tabular}{|c|c|c|c|c|c|c|c|}
\hline & P4 & E193 & E194 & E195 & E196 & E197 & E199 \\
\hline 16.11 & 13.3 & 507.1 & 921.5 & 828.7 & 994 & 794.5 & 1090 \\
\hline 17.11 & 14.6 & 524.1 & 1307.2 & 982.5 & 1303.5 & 864.5 & 1291.9 \\
\hline 18.11 & 13 & 470.5 & $\mathbf{1 0 8 5 . 7}$ & 821.2 & 918.5 & 670.8 & 903.6 \\
\hline
\end{tabular}

Table 2. Summary of average data rate per $1 \mathrm{sec}$. frame

\begin{tabular}{|c|c|c|c|c|c|c|c|}
\hline & P4 & E193 & E194 & E195 & E196 & E197 & E199 \\
\hline 16.11 & 1568 & 2920 & 5080 & 4504 & 4592 & 4728 & 5496 \\
\hline 17.11 & 2232 & 3984 & 8392 & 7616 & 6168 & 6560 & 7080 \\
\hline 18.11 & 1936 & 3152 & $\mathbf{5 7 2 8}$ & 4720 & 5208 & 5520 & 5040 \\
\hline
\end{tabular}

Table 3. Summary of maximum data rate per $1 \mathrm{sec}$. frame

Eventually we have selected RADAR E-194 as a representative one and the data from the $18^{\text {th }}$ as an average amount of data to be transmitted. You can see (shadowed) the average and the peak data rate, remarking the big difference between them.

\section{Modem design}

Once we have selected an average scenario, we proceed to describe the main features of our design. We started from a previous design for OFDM transmission and as possible, we intended to maintain some of the strategies that have shown to provide satisfactory performance as pilot density, synchronization, channel estimation and interpolation and intercarrier spacing $(37.5 \mathrm{~Hz})[5,7-9]$. Other figures as the number of carriers and modulations have been selected specifically for this design. Next table shows some examples of suitable designs:

\begin{tabular}{|c|c|c|c|c|c|c|c|c|c|}
\hline$N_{\text {carr }}$ & \multicolumn{2}{|c|}{100} & \multicolumn{2}{c|}{150} & \multicolumn{2}{c|}{200} \\
\hline$N_{\text {symb }}$ & \multicolumn{3}{|c|}{20} & \multicolumn{2}{c|}{20} & \multicolumn{3}{c|}{20} \\
\hline BW [Hz] & \multicolumn{2}{|c|}{4537.5} & \multicolumn{2}{c|}{$\mathbf{6 7 8 7 . 5}$} & \multicolumn{3}{c|}{9037.5} \\
\hline M-QAM & 4 & 16 & 64 & $\mathbf{4}$ & $\mathbf{1 6}$ & $\mathbf{6 4}$ & 4 & 16 & 64 \\
\hline $\begin{array}{c}\text { Data rate } \\
{[\mathrm{Kb} / \mathrm{s}]}\end{array}$ & 4 & 8 & 12 & $\mathbf{6}$ & $\mathbf{1 2}$ & $\mathbf{1 8}$ & 8 & 16 & 24 \\
\hline
\end{tabular}

Table 4. Different modem designs

According to tables 4 and 3 we may make the decision about the bandwidth evaluating the percentage of usage of different M-QAM modulations.

\begin{tabular}{|c|ccc|ccc|ccc|}
\hline $\begin{array}{c}\text { M- } \\
\text { QAM }\end{array}$ & 4 & 16 & 64 & 4 & 16 & 64 & 4 & 16 & 64 \\
\hline $\begin{array}{c}\text { Data } \\
\text { rate } \\
{[\mathrm{Kb} / \mathrm{s}]}\end{array}$ & 4 & 8 & 12 & 6 & 12 & 18 & 8 & 16 & 24 \\
\hline $\begin{array}{c}\% \\
\text { frames }\end{array}$ & 63.1 & 32.44 & 4.226 & 86.7 & 13.07 & 0.23 & 95.54 & 4.45 & 0.0067 \\
\hline
\end{tabular}

Table 5. Percentage of usage of different modulations

Analysing tables 4 and 5, we concluded that 150 carriers spanning around $6.8 \mathrm{KHz}$ will require around $86 \%$ a 4-QAM (very robust), some 13\% a 16-QAM and 64-QAM nearly never.

\subsection{Block diagram}

This section will describe the block diagram of both transmitter and receiver. Main aspects will be described in the sequel for clarity.

Figure 2 shows the structure of transmitter. The system works as follows:

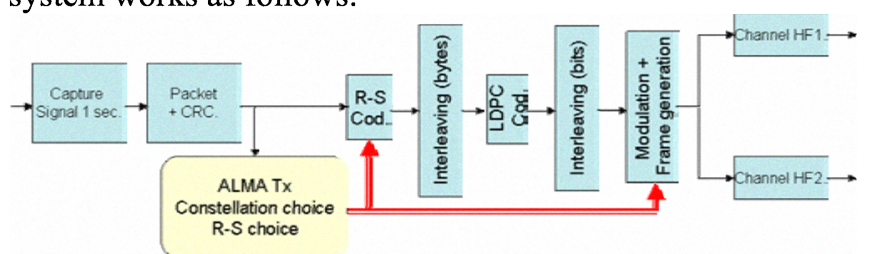

Figure 2. Block diagram of transmitter 
a) We capture data from the RADAR source during 1 second counting from the first plot. At that moment, the time is set to zero until a new plot appears that triggers the count again. This way we are sure that maximum delay is under the limit.

b) ASTERIX data do not have all the same length but we have made a study about the distribution of the number of bytes and the mode is 18 . So, we have defined a packet of this size. If the plot is shorter, we add zeros, if it is longer, it occupies two packets. We add a 16 bits CRC tail to have a clear indicator of correct plot.

c) Depending on the amount of data to be transmitted, the transmitter manager (ALMA-Tx) makes the decision of the required constellation size and also the amount of available redundancy.

c) FEC coding is a concatenated structure where the inner code is a LDPC $1 / 2$ and the outer code is a shortened ReedSolomon. Interleavers at byte and bit level are also included.

d) Once the frame is constructed appending the header - data field and ending flag, the same signal is transmitted by several channel is parallel (two in the figure and also in the real link).

At the receiver side we considered two options. First one intended to make a combination at signal level using MRC (Maximum Ratio Combining) principles as is shown in figure 3 .

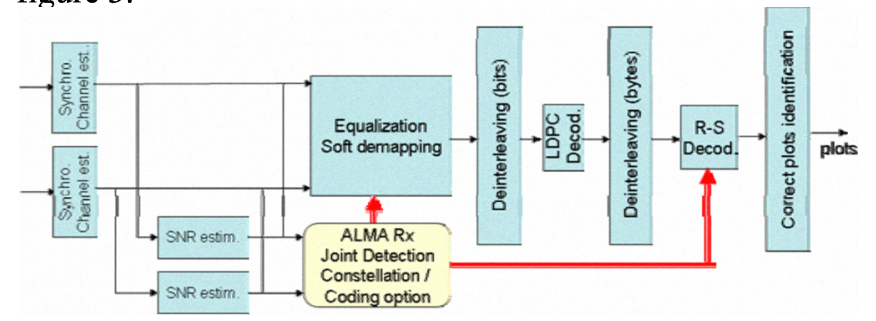

Figure 3. Receiver based on joint equalization

However, this structure was rejected after some simulations because the performance in realistic conditions was far away of the expected ones. The main reason was the difficulty of estimating the SNR (recall that frames are very short) accurately and properly weighting the combination of the MRC structure. We observed that in many cases, one of the channels was very biased in the channel estimation therefore degrading the joint detection much more than in the case where the other channel were detected independently. So we decided a different architecture, more complex but with a more reliable indicator of quality: the $\mathrm{CRC}$ indicator.

Figure 4 shows the block diagram where it can be observed that after proper synchronization, we demodulate the plots at both branches. All procedures are essentially the opposite of the transmitter processes. The receiver manager (ALMA-Rx) extracts the side information (Reed-Solomon code, number of padding zeros, constellation size) and defines the demodulation process. Once we have both CRCs, we choose the one that is correct.

\subsection{Coding process}

Coding is used for two purposes. On one hand, a CRC code is proposed for correctness indicator. We have implemented the
CRC-CCIT with 2 bytes of redundancy because it is well known that works very satisfactorily.

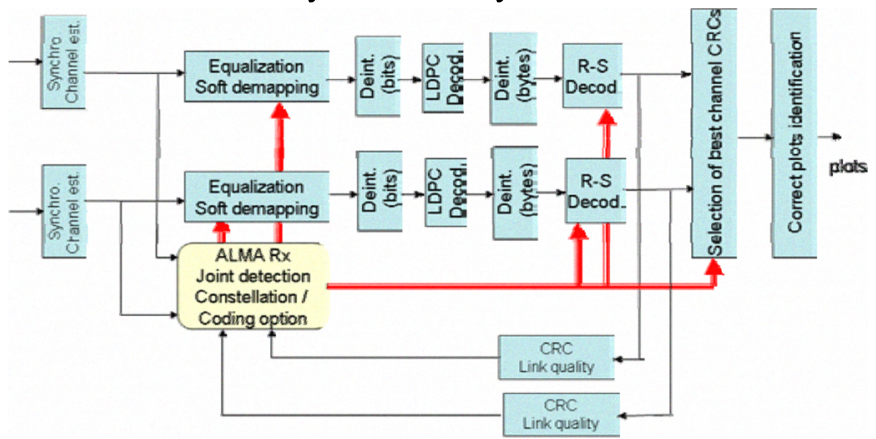

Figure 4. Receiver with combination at plot level

Regarding the FEC, we propose a concatenated structure with an inner LDPC (1/2) code and an outer code using Reed-Solomon (255-237). Two interleavers, one at bit level and the other at byte level (due to the Reed-Solomon) are used to randomize errors. However, we have realized that their role in this application is not very satisfactory due to the lack of time diversity. In the future, we have in mind to improve this architecture adding time-frequency coding and interleaving to improve the performance using also the frequency diversity of the multichannel transmission.

The Reed-Solomon selection, depending on the available redundancy is based on a shortening process $\mathrm{R}-\mathrm{S}(n, k)$ where $n-k=255-237=18$ bytes.

\subsection{ALMA Tx /Rx functionality}

The main tasks of the ALMA-Tx are:

a) Timing control to trigger the transmission process when the delay reaches the limit.

b) Selection of the modulation size and packing the plots including the CRC tail.

c) Selection of the Reed-Solomon size according to the available redundancy.

d) Code all the side information in the header regarding the constellation size, number of zeros and shortening process.

The main tasks for the ALMA Rx are basically the opposite of the former ones:

a) After proper synchronization, extract the side information from the header and set up the receiver accordingly.

b) Selection of the correct plot depending on the CRC. To save computation costs, in fact, only one branch is demodulated (the one with better SNR) and the other is only demodulated when one plot is found incorrect).

c) Only the correct ones are presented on the screen.

\section{Simulated results}

Our system was initially tested using a simulator channel to check out how it works and define some open issues that were not very clear at the initial stage as the coding selection. This section just presents some of these results, including some preliminary analysis using just one channel and some later results using two parallel transmissions. The channel simulator follows the Watterson model and the multichannel 
assumes independent realizations. In principle, both channels are at the same SNR, although we have realized that this is far away of real transmissions not only due to the differences between the propagation paths but more importantly the interference level.

\subsection{Single channel transmission}

Figure 5 shows one interesting result where we wanted to check the effect of the variable-rate coding. We compare the BER of random data using just LDPC and 4-QAM and separately the same random data using 16-QAM. On the other hand, we present the ASTERIX data with the concatenated FEC structure. The performance is very close to the pure 4QAM transmission showing the powerful effect of the FEC.

\subsection{Multiple channel transmission}

Figure 5 also shows the gain of including the frequency diversity (labelled as MC- MultiChannel) in front of the previous Single Channel (SC). A gain of some $2 \mathrm{~dB}$ is achieved.

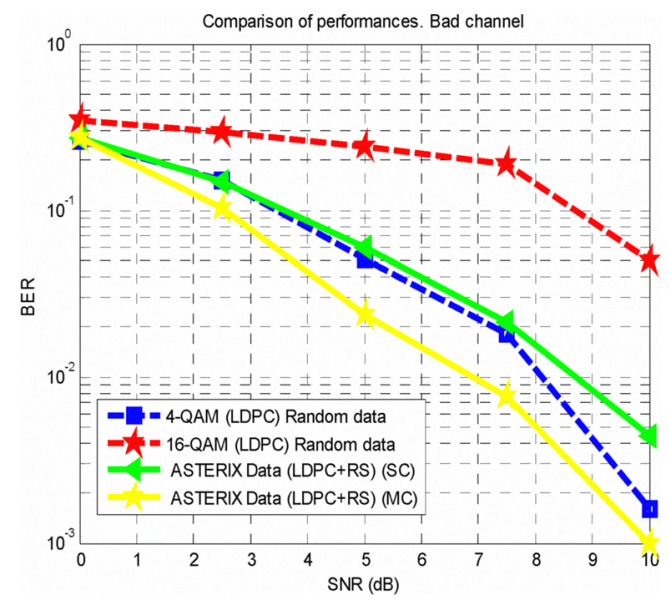

Figure 5. Simulated Multiple channel transmission

Figure 6 presents the results in terms of the Plot Error Rate (PIER), which is the main figure to characterize the performance of the system. It is shown, for comparative purposes the Single Channel and the Multiple Channel, splitting the performance for the frames using 4-QAM, those using the 16-QAM and the global scenario. Eventually, we are guessing that we can reach a $\mathrm{PlER}=610^{-3}$ at $\mathrm{SNR}=10 \mathrm{~dB}$. Clearly this figure is very optimistic and in the real link we will not be able to reach it, at least under the testing conditions.

\section{Real link results}

Real tests have been very complicated because we needed to implement and test our own receiver in order to cope with broader single channel transmission (around $7 \mathrm{KHz}$ ) and simultaneous transmissions separated tenths or hundreds of $\mathrm{KHz}$. The description of this transceiver is out of the scope of this paper and in fact will be presented in a full paper in this conference [6].

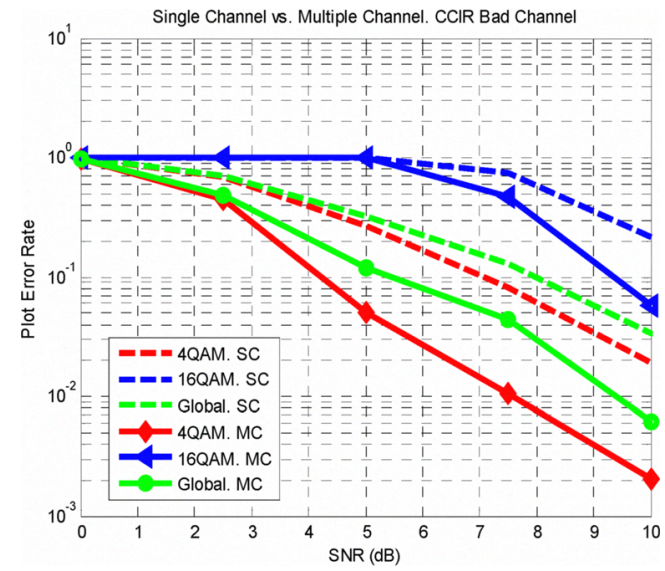

Figure 6. Plot Error Rate. Different Configurations

\subsection{Description of the testing procedure}

The results of these tests must be considered as preliminary because our facilities and conditions have many limitations. Our intention has been to repeat a transmission of around 20 minutes, several times per day, changing the frequency separation of the multichannel transmission. The link is between Las Palmas de Gran Canaria and Madrid in the 14 $\mathrm{MHz}$ band to make sure that we have an open link most of the day. However, as this is a free band, we have suffered from many interferences that have degraded the SINR very much. Also, some problems with the broadband amplifier have limited our transmitter power. In practice, what we have done is to monitor the occupancy of several channels before the transmission and select the cleanest. Unfortunately, many times, after some interval, a strong interference degraded the performance.

We have tested two main scenarios, one where the two channels were nearby, separated some $36 \mathrm{KHz}$ and other test with channel separated 324 and $204 \mathrm{KHz}$ (close to the bandwidth of our antenna) to test the diversity gain. Next sections will show some results.

\subsection{Transmission by nearby channels}

Table 6 shows the BER and PIER results of tests 1-5 carried out at different hours in 2008 , November the $26^{\text {th }}$, channel one tuned at $14.248 \mathrm{MHz}$ and channel 2 at $14.212 \mathrm{MHz}$.

\begin{tabular}{|c|c|c|c|}
\hline Test & BER Ch. 1 / Ch. 2 & Joint BER & PIER \\
\hline 1 & $0.1555 / 0.3330$ & 0.1670 & 0.4242 \\
\hline 2 & $0.1004 / 0.1054$ & 0.0537 & 0.2084 \\
\hline 3 & $0.1126 / 0.2274$ & 0.1086 & 0.3214 \\
\hline 4 & $0.2141 / 0.2495$ & 0.1941 & 0.5459 \\
\hline 5 & $0.1278 / 0.1217$ & 0.0995 & 0.2324 \\
\hline
\end{tabular}

Table 6. Performance nearby channels

We can observe that the variations from test to test are very significant and in fact the best performance makes the PIER around $20 \%$. 


\subsection{Transmission by far away channels}

Table 7 shows the results when using different separations between the channels. Tests 6-8 used two channels at 14.362 $\mathrm{MHz}$ and $13.988 \mathrm{MHz}$, while tests 9-11 used $14.342 \mathrm{MHz}$ and $14.138 \mathrm{MHz}$.

\begin{tabular}{|c|c|c|c|}
\hline Test & BER Ch. 1 / Ch. 2 & Joint BER & PIER \\
\hline 6 & $0.0723 / 0.2123$ & 0.0858 & 0.2639 \\
\hline 7 & $0.0628 / 0.2735$ & 0.0856 & 0.2123 \\
\hline 8 & $0.0816 / 0.1116$ & 0.0491 & 0.1594 \\
\hline 9 & $0.0700 / 0.1091$ & 0.0402 & 0.1486 \\
\hline 10 & $0.1195 / 0.2051$ & 0.0956 & 0.300 \\
\hline 11 & $0.0816 / 0.1116$ & 0.0461 & 0.1476 \\
\hline
\end{tabular}

Table 7. Performance far away channels

It can be concluded that having more frequency diversity, we may have a better performance reaching PlERs around $15 \%$. Although we are clearly we are far away of the $0.6 \%$ obtained from the simulated environment, we may say that for further analysis we can be optimistic if we able to use more professional facilities as those belonging to Aena. In that case, increasing the SINR transmitting with more power and over reserved channels (nearly interference free), we may close up to the simulated results.

\subsection{Snap-shot of a RADAR screen}

We should not forget the nature of this transmission and the high correlation among the data in different frames. Clearly, every turn of the antenna will point to a very similar scenario with high correlation in space and time, especially in civil aviation. This is well known by RADAR experts that are able to reconstruct a track even if some plots are lost. Figure 7 shows a snapshot of test 11 after some 200 frames representing the transmitted plot in green circle and the received one in blue $\mathrm{x}$. We have shown these results to professionals in Air Traffic Control and it seems that performance could be acceptable, for some of the tests.

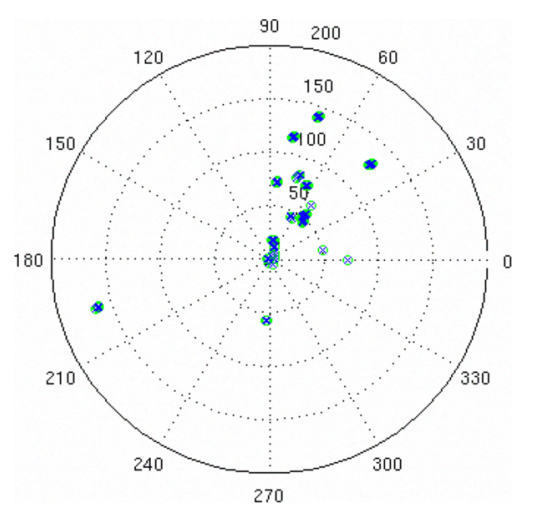

Figure 7. Snap shot of the RADAR screen. Test 11

\section{Conclusions}

This paper shows a preliminary real tests where we have designed an OFDM modem specifically for data RADAR transmission fulfilling very restricted delay conditions. We have overcome this requirement along with the unidirectional transmission by a powerful coding scheme and a dual transmission by separated channels to retrieve frequency diversity to compensate the short time diversity. The main goal of this approach is to verify these ideas in a real link between Madrid and Las Palmas de Gran Canaria. We have run several links for different channelizations and daytime hours.

\section{Acknowledgements}

We would like to thank Aena for their support and also the Spanish Education and Science Ministry under Grant TEC2007-67520-C02-01/02/TCM

\section{References}

[1] Eurocontrol Standard Document for Surveillance Data Exchange, Part 1, All Purpose Structured Eurocontrol Surveillance Information Exchange (ASTERIX), SUR.ET1.ST05.2000-STD-01-01, Feb. 2002.

[2] Eurocontrol Standard Document for Radar Data Exchange, Part 2a, Transmission of Monoradar Data, Target Reports, SUR.ET1.ST05.2000-STD-02a-01, Aug. 2002.

[3] Eurocontrol Standard Document for Radar Data Exchange, Part 2b, Transmission of Monoradar Service Messages, SUR.ET1.ST05.2000-STD-02b-01, Nov.1997

[4] National Areas assignment. http://www.eurocontrol.be/ asterix/public/standard page/sac list.html

[5] Pérez-Álvarez, I.; López-Pérez, J.; Zazo-Bello, S.; Raos, I.; Pérez-Díaz, B.; Jiménez-Yguacel, E. Real Link of a High Data Rate OFDM Modem: Description and Performance. IRST 2009: 11th International Conference on Ionospheric Radio Systems \& Techniques.Edinburgh, U.K.; April 2009

[6] Pérez-Díaz, B.; Jiménez-Yguacel, E.; López-Pérez, J.; Pérez-Álvarez, I.; Zazo-Bello, S.; Medieta-Otero, E. Design and construction of a broadband $(1 \mathrm{MHz})$ digital $\mathrm{HF}$ transceiver for multicarrier and multichannel modulations. IRST 2009: 11th International Conference on Ionospheric Radio Systems \& Techniques.Edinburgh, U.K.; April 2009.

[7] Santana, H.; Zazo, S.; Pérez, I.; Raos, I.; Mendieta, E.; López, J. Validation of a HF Spread Spectrum MultiCarrier Technology through real Link Measurements. European Trans. on Telecommunications (ETT), Vol17,Nov.-Dec. 2006 [8] Santana-Sosa, H.; Pérez-Álvarez, I.; A.; Mendieta-Otero, E.; López-Pérez, J.;Zazo, S.; Raos, I.; Performance over a real link of a HF software radio modem for interactive digital voice communications. HF 2006: Tenth International Conference on HF Radio Systems and Techniques 2006, London, UK, July 2006

[9] Zazo, S.; F. Bader; J.M Paez Borrallo. Spread Spectrum OFDM Modem for HF Voice-Band Link Over Fading Channels. European Transactions on Telecommunications, Vol.11, Nov.-Dec. 2000 